\title{
Interpreting COVID-19 Test Results: a Bayesian Approach
}

$\mathrm{J}$ Gen Intern Med 35(8):2490-1

DOI: $10.1007 / \mathrm{s} 11606-020-05918-8$

(c) Society of General Internal Medicine 2020

\section{INTRODUCTION}

As physicians care for patients with contact history and symptoms that might represent coronavirus disease 2019 (COVID19), interpreting the results of polymerase chain reaction (PCR) assays from nasal and pharyngeal swabs is crucial. While a positive result in an acutely ill patient is straightforward, how should physicians interpret negative tests in patients with suspected COVID-19 infection?

Physicians and patients often place inappropriate confidence in test results, even when those tests are imperfect. ${ }^{1}$ Specifically, physicians may minimize their own clinical reasoning (e.g., their pre-test probability of disease) and defer to a test result that may not be correct. With PCR testing for COVID-19, false negative tests are particularly concerning, potentially leading to an inappropriate sense of security regarding infectivity.

To accurately interpret test results, one needs to know the positive and negative predictive values of a test in the setting applied, which depend on its sensitivity and specificity, along with prevalence or pre-test probability. Although the specificity of PCR assays for COVID-19 appears to be close to 100\%, documenting its sensitivity is surprisingly elusive. ${ }^{2}$ Realworld sensitivity of the COVID-19 assay is especially impacted by difficulty in sampling technique for obtaining specimens using nasopharyngeal swabs. ${ }^{3}$ One recent unpublished (nonpeer reviewed) study based on the experience in China suggested a sensitivity of $70 \%{ }^{4}$

We believe that Bayes theorem can be applied to the interpretation of negative PCR results in patients with suspected COVID-19 infection. To illustrate, we simulate two patient scenarios with differing contact history and clinical presentations.

\section{METHODS}

We applied a Bayesian analysis to interpret negative and positive COVID-19 PCR assay results for two clinical scenarios. For both scenarios, we assumed a PCR assay specificity of $99.9 \%$ and varied the sensitivity from 70 to $90 \% .^{4,5}$

Scenario 1 (high pre-test probability of COVID-19 infection): A 32 -year-old nurse presents with 2 days of fevers to $102^{\circ}$,

Received April 22, 2020

Accepted May 6, 2020

Published online June 3, 2020 cough, and subjective dyspnea. She works in an emergency room that has evaluated numerous COVID-19 patients. She reports using appropriate personal protective equipment. We estimated a pre-test probability of COVID-19 infection at $90 \%$ (but varied it to as low as $70 \%$ ).

Scenario 2 (low pre-test probability of COVID-19 infection): A 25-year-old male presents with subjective fevers (no temperature taken), cough, and subjective dyspnea. He has no significant exposures but lives where COVID-19 infections were reported; he has worked at home for the past month with occasional shopping for food. He reports frequent hand washing and practices social distancing. We estimated a pre-test probability of COVID-19 infection at 5\% (but varied it to as high as $10 \%$ ).

\section{RESULTS}

For the high-risk scenario with our estimated 90\% pre-test probability, the post-test probability of a false negative test ranged from 47 to $73 \%$ (Table 1). With a $70 \%$ pre-test probability, the post-test probability of a false negative ranged from 19 to $41 \%$. For a low-risk scenario with a pre-test probability of 5-10\%, the post-test disease probability with a negative test ranged from 0.5 to $3.2 \%$. Disease likelihood with a positive test remained $>99.9 \%$ in the high-risk scenario and $>97.4 \%$ in the low-risk patient.

\section{DISCUSSION}

We applied a Bayesian approach to illustrate the interpretation of COVID-19 negative tests based on the clinical suspicion of disease probability. A positive test in both high pre-test and low pre-test scenarios most likely represents acute infection. Likewise, a negative test in a low pre-test probability case

Table 1 Estimates for Post-Test Probability of Acute COVID-19 Infection for Simulated Patient Scenarios

\begin{tabular}{lllll}
\hline \hline $\begin{array}{l}\text { Clinical } \\
\text { Scenarios }\end{array}$ & $\begin{array}{l}\text { Pre-test } \\
\text { probability } \\
(\%)\end{array}$ & $\begin{array}{l}\text { PCR assay } \\
\text { sensitivity } \\
(\%)\end{array}$ & $\begin{array}{l}\text { Post-test probability } \\
\text { of acute COVID-19 } \\
\text { infection }\end{array}$ \\
\cline { 3 - 5 } & & & $\begin{array}{l}\text { Positive } \\
\text { test }(\boldsymbol{\%})\end{array}$ & $\begin{array}{l}\text { Negative } \\
\text { test }(\boldsymbol{\%})\end{array}$ \\
\hline Patient 1: & 70 & 70 & 100 & 41.2 \\
high pre- & & 90 & 100 & 18.9 \\
test proba- & 90 & 70 & 100 & 73.0 \\
bility & \multirow{2}{*}{5} & 90 & 100 & 47.4 \\
Patient 2: & \multirow{2}{*}{5} & 70 & 97.4 & 1.6 \\
low pre-test & & 90 & 97.9 & 0.5 \\
probability & 10 & 70 & 98.7 & 3.2 \\
& & 90 & 99.0 & 1.1 \\
\hline
\end{tabular}


indicates a low likelihood of acute infection. However, when COVID-19 infection is likely, such as in a healthcare worker with significant exposure, a negative test should not rule out acute infection. In this case, as recommended by the $\mathrm{CDC}{ }^{6}$ repeat testing or further evaluation should be considered.

Chester B. Good, MD, MPH ${ }^{1,2,3}$

Inmaculada Hernandez, Pharm $\mathrm{D}, \mathrm{PhD}^{3,4}$

Kenneth Smith, MD, MS ${ }^{2}$

${ }^{1}$ Centers for High Value Health Care and Value-Based Pharmacy Initiatives Insurance Services Division, UPMC Health Plan,

600 Grant Street, Pittsburgh, PA 15219, USA

${ }^{2}$ Division of General Internal Medicine, University of Pittsburgh, School of Medicine,

Pittsburgh, PA, USA

${ }^{3}$ Center for Pharmaceutical Policy and Prescribing, University of Pittsburgh, School of Medicine,

Pittsburgh, PA, USA

${ }^{4}$ Department of Pharmacy and Therapeutics, University of Pittsburgh, School of Pharmacy, Pittsburgh, PA, USA

Corresponding Author: Chester B. Good, MD, MPH; Centers for High Value Health Care and Value-Based Pharmacy Initiatives Insurance Services Division, UPMC Health Plan 600 Grant Street, Pittsburgh, PA 15219, USA (e-mail: Goodcb2@upmc.edu).

\section{Compliance with Ethical Standards:}

Conflict of Interest: The authors declare that they do not have a conflict of interest.

\section{REFERENCES}

1. Jha S. False Negative: COVID-19 Testing's Catch-22 - And the consequences of being wrong. Medpage Today, Mar 31 2020; https://www. medpagetoday.com/infectiousdisease/covid19/85717.

2. If You Have Coronavirus Symptoms, Assume You Have the Illness, Even if You Test Negative. New York Times, Apr 1 2020; https://www.nytimes. com/2020/04/01/well/live/coronavirus-symptoms-tests-false-negative. html?smid=nytcore-ios-share Accessed Apr 112020.

3. Cummins S. Why the Coronavirus Test Gives So Many False Negatives. Slate, Apr 6 2020; https://slate.com/technology/2020/04/coronavirustesting-false-negatives.html Accessed Apr 112020.

4. Yang $\mathbf{Y}$, Yang $\mathbf{M}$, Shen $\mathbf{C}$ et al. Evaluating the accuracy of different respiratory specimens in the laboratory diagnosis and monitoring the viral shedding of 2019-nCoV infections. doi: https://doi.org/10.1101/2020.02. 11.20021493

5. Centers for Disease Control and Prevention. 2019-Novel Coronavirus (2019-nCoV) Real-Time RT-PCR Diagnostic Panel. Mar 32020 https:// www.fda.gov/media/134922/download Accessed Apr 112020.

6. Centers for Disease Control and Prevention. COVID-19 Fact Sheet for Healthcare Providers. Mar 152020 https://www.cdc.gov/coronavirus/ 2019-ncov/downloads/Factsheet-for-Healthcare-Providers-2019-nCoV. pdf Accessed Apr 112020.

Publisher's Note: Springer Nature remains neutral with regard to jurisdictional claims in published maps and institutional affiliations. 